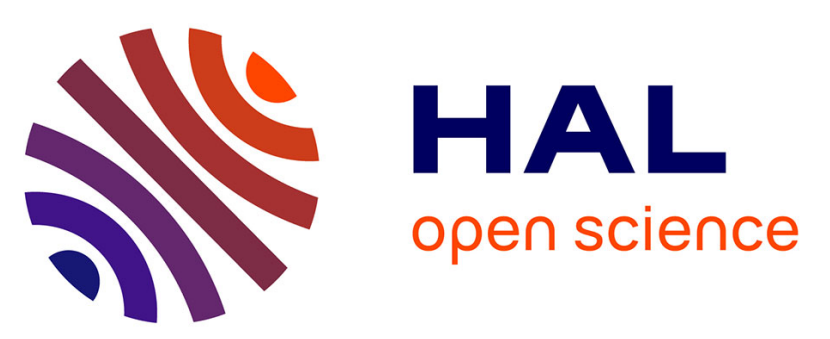

\title{
The role of dispersion forces in metal-supported self-assembled monolayers
}

\author{
Roberto A. Boto, J. Contreras-García, M. Calatayud
}

\section{To cite this version:}

Roberto A. Boto, J. Contreras-García, M. Calatayud. The role of dispersion forces in metal-supported self-assembled monolayers. Computational and Theoretical Chemistry, 2015, 1053, pp.322-327. 10.1016/j.comptc.2014.10.015 . hal-01102760

\section{HAL Id: hal-01102760 \\ https://hal.sorbonne-universite.fr/hal-01102760}

Submitted on 13 Jan 2015

HAL is a multi-disciplinary open access archive for the deposit and dissemination of scientific research documents, whether they are published or not. The documents may come from teaching and research institutions in France or abroad, or from public or private research centers.
L'archive ouverte pluridisciplinaire HAL, est destinée au dépôt et à la diffusion de documents scientifiques de niveau recherche, publiés ou non, émanant des établissements d'enseignement et de recherche français ou étrangers, des laboratoires publics ou privés. 
The role of dispersion forces in metal-supported self-assembled monolayers

\author{
Roberto. A. Boto ${ }^{1,2,3}$, J. Contreras-García ${ }^{1,2}$, M. Calatayud ${ }^{1,2,4^{*}}$ \\ ${ }^{1}$ Sorbonne Universités, UPMC Univ. Paris 06, UMR 7616, Laboratoire de Chimie Théorique, F-75005, \\ Paris, France \\ ${ }^{2}$ CNRS, UMR 7616, Laboratoire de Chimie Théorique, F-75005, Paris, France \\ ${ }^{3}$ Sorbonne Universités, UPMC Univ. Paris 06, ICS , F-75005, Paris, France \\ ${ }^{4}$ Institut Universitaire de France
}

Corresponding author:

Dr. M. Calatayud

Laboratoire de Chimie Théorique Case 137

Université P. M. Curie

75252 Paris

e-mail : calatayu@lct.jussieu.fr

phone : +33144272505

fax : +33144274117 


\section{Abstract}

The self-assembly of organic molecules (octylamine) is studied by means of periodic DFT calculations including Grimme D2 dispersion scheme. It has been found that in the gas-phase dispersive forces are crucial when packing is considered, modifying considerably the potential energy surface compared to the case of a molecular dimer. In interaction with a metallic surface (platinum), the electronic interaction between adsorbate and substrate is predominant, dispersion forces mainly increasing the adsorption strength. A detailed study on the structural parameters of the moleculemetal system is reported to gain understanding on the factors affecting their stability. The intermolecular bonding in these systems is analyzed by means of the non-covalent interactions $\mathrm{NCl}$ index. The decomposition of the 2D packing along each direction $(x, y)$ highlights the importance of the diagonal contributions in the stabilization of the square pattern. Moreover, the stabilizing nature of $\mathrm{H}-\mathrm{H}$ interactions between neighboring molecules is supported in this model.

Keywords: SAM, DFT, NCl, platinum, amine 


\section{Introduction}

The binding of long alkyl chain molecules to metal surfaces is being deeply investigated for application in many technological fields such as films, electronic junctions, tribology or coating [1]. The stability of such systems is mainly due to a competition between metal-molecule and moleculemolecule interactions, whose strength critically determines their physicochemical properties. For instance, in gold/thiol self-assembled monolayers (SAMs), the surface-molecule interaction must be strong to immobilize the alkyl chains on the metal surface $[1,2]$. Another example is the synthesis of metal nanoparticles in mild conditions, where the alkylamine chains are used as surfactant to stabilize the metallic surface $[3,4]$. Here, the metal-surface strength must be intermediate: if it is too strong, the molecule blocks the surface site and the particle cannot grow further leading to small nanocrystals, but if it is too weak the particle surface is not stabilized and it grows isotropically leading to large spherical nanoparticles without control on the size or shape. Understanding the nature and the strength of the interactions between surfaces and long chain molecules is thus of great importance and may help in the design of systems with a specific molecule-surface and molecule-molecule strengths.

The intermolecular interactions in SAMs come from the vicinity of the neighboring molecules that form an ordered two-dimensional layer and are mainly caused by dispersion forces. The role of the dispersion forces between the alkyl chains is poorly understood although it may become crucial in the stabilization of the system. Dispersion forces are commonly estimated from different theoretical approaches. Semi-hybrid functionals have been used to study thiol/gold SAMs [5]. Recently, the implementation of the Grimme approach $[6,7]$ in solid state codes has allowed exploring their role in adsorption systems. It has been found that the inclusion of Grimme DN corrections can significantly affect the calculated geometry of adsorption as well as its strength [8]. Also, the self-assembling of polymers in vacuo has been successfully described by using such approach [9]. In the last years, dispersion in short alkyl chains assembly on metals has been studied with ab initio methods $[10,11]$. 
As regards bonding analysis on SAMs, the reactivity of the tail groups has been studied by conceptual DFT methods [12] and the chemical bond between surfactant and surface studied with the electron localization function ELF [13]. However, little is known about the weak interactions involved in the packing of molecules, as in self-assembled monolayers on a solid surface. The aim of the present paper is to highlight the crucial role of dispersion forces in the organization of long alkyl molecules in two dimensions. It will be shown that in the gas-phase the energetic profile of a packing of molecules is dramatically affected by such interactions. Standard Density Functional Theory (DFT) calculations are used combined with Grimme D2 approach. A bonding analysis based on Non Covalent Interactions $(\mathrm{NCl})$ index is applied to illustrate the nature of the intermolecular interactions.

\section{Models and methods}

The platinum (100)-octylamine $\left(\mathrm{NH}_{2} \mathrm{C}_{8} \mathrm{H}_{17}\right)$ system has been chosen because it has been recently reported to lead to stable cubic platinum nanoparticles $[3,14]$. Such particles are synthesized in mild conditions at the interface between an organic and an aqueous phase. The octylamine is used as surfactant to stabilize the metal particle in the organic (toluene) phase. The particles obtained are cubic and the metal will be modeled by a (100)-terminated five layers-thick slab. The octylamine is investigated in the gas-phase as well as supported on the platinum slab in order to decompose the parameters affecting stability: molecule-molecule distance, molecule packing, surface-molecule distance and coverage.

The calculations are carried out with the revised Perdew Burke Ernzerhof $[15,16]$ functional as implemented in the VASP code $[17,18]$. Test calculations on the Pt fcc bulk lattice parameter and cohesive energy are shown in Figure S1, they support the choice of rPBE+D2 level of theory. Projector Augmented Wave (PAW) pseudopotentials $[19,20]$ combined to plane waves (cutoff $=400 \mathrm{eV}$ ) represent the electronic distribution. An appropriate set of k-points is used to sample the Brillouin zone. Periodic boundary conditions apply and a vacuum of at least $15 \AA$ is included to avoid 
interaction between successive layers. Starting from the gas-phase optimized geometry at the rPBE level, calculations are done without geometrical optimization for the gas-phase studies and with the conjugate-gradient algorithm for the surface-molecule interaction studies, with the geometrical constrains specified in each case (see Table S1). The interaction energies are calculated by the formula:

$$
A+B=[A B] \text { where } E_{i n t}=E_{[A B]}-E_{A}-E_{B}
$$

thus negative for exothermic reactions.

Dispersion interactions are included by using the Grimme D2 approach as implemented in the VASP code (parameters for Pt: radius=1.676 $\AA$ and $C_{6}=19.46 \mathrm{Jnm}^{6} \mathrm{~mol}^{-1}$ obtained from [7]). As noticed in the literature [8], the problem of metal screening of dispersive forces can be roughly corrected by including dispersion only on the uppermost slab layer. We have considered this possibility together with the pure rPBE and full D2, denoted as $1 \mathrm{~L}, \mathrm{rPBE}$ and $\mathrm{D} 2$ respectively. Tests on the propilamine dimer potential energy curve have been carried out at the MP2/6-311++g(3df,2pd), BSSE corrected, together with the rPBE and rPBE+D2 levels, and show very good agreement with the rPBE+D2 setting used in the present work, as can be seen in Figure S2.

It has been recently shown that although the energetic balance at weak interactions is cumbersome to detect from energetics, the density $(\rho)$ reconstruction by those methods is accurate enough to reveal the interactions present in the system. This is the principle lying behind the recently reported index for detecting Non-Covalent-Interactions, $\mathrm{NCl}$ [21]. This index enables the identification and characterization of weak interactions of various strengths as chemically intuitive reduced density gradient isosurfaces that reveal both stabilizing (hydrogen bonds, van der Waals) and destabilizing (steric clashes) interactions. It can also be applied to ionic interactions [22]. In order to achieve a better comprehension of the nature of bonding and packing, representative systems will be studied by means of the $\mathrm{NCl}$ method. The $\mathrm{NCl}$ analysis has been carried out with the critic2 code [23] and visualized in VMD [24]. 


\section{Results and discussion}

We have chosen to investigate independently the key factors in the stabilization of the system surface-molecule so we can derive the nature and the strength of the forces responsible for each interaction. Thus, we have computed the gas-phase molecule-molecule interaction as regards their interatomic distance, the relative orientation and the packing effect. Then, we have studied the surface-molecule interaction concerning the adsorption site, the distance surface-molecule, the tilting angle and the coverage.

\subsection{The molecule-molecule interaction in the gas-phase}

The potential energy surface of an octylamine dimer in the gas-phase is presented in Figure 1. It can be seen that the curve decays asymptotically and reaches a minimum for a distance of $5.5 \AA$ ( $\mathrm{rPBE}$ ) and $5.0 \AA$ (D2). The energy gain with respect to the isolated molecule in the gas-phase is almost athermic for rPBE and only slightly exothermic, $-0.12 \mathrm{eV}$, for D2. Compared to the propilamine results (Figure S2), the energy gain is of $0.05 \mathrm{eV}$. Such gain is due to the longest alkyl chain that increases the dispersive interactions.

The packing effect of the molecule on the surface has been calculated by considering the molecule in a tetragonal unit cell repeated in three dimensions. The $a$ parameter is modified so as to consider the distance between consecutive neighboring molecules. The curves obtained, presented in Figure 1, show a behavior similar to that of the dimer for the pure rPBE functional. Instead, a qualitative change is observed for the D2 calculations: a minimum in the curve appears at a distance of $4.5 \AA$, with an energy gain of $-0.28 \mathrm{eV}$. Therefore, dispersion forces seem to be crucial for representing packing in two-dimensions in the gas-phase. 
Gas-phase dimer

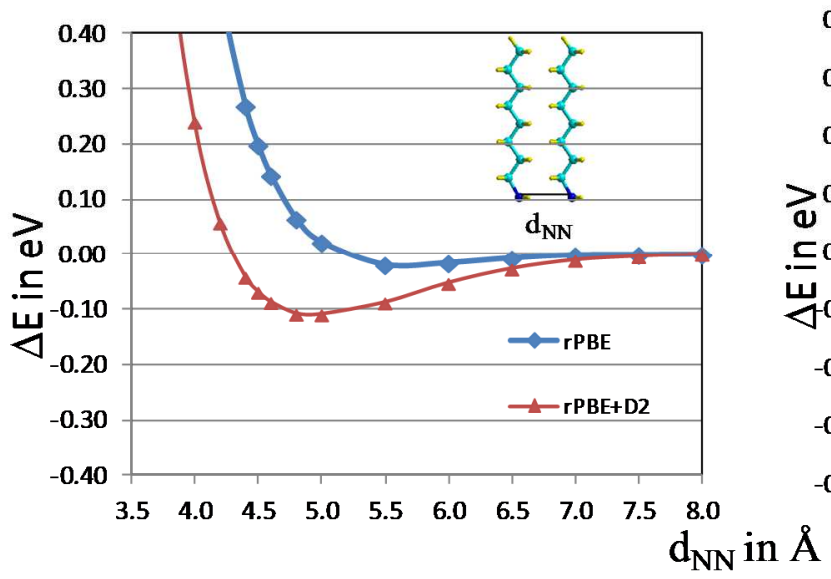

Gas-phase 2D square packing

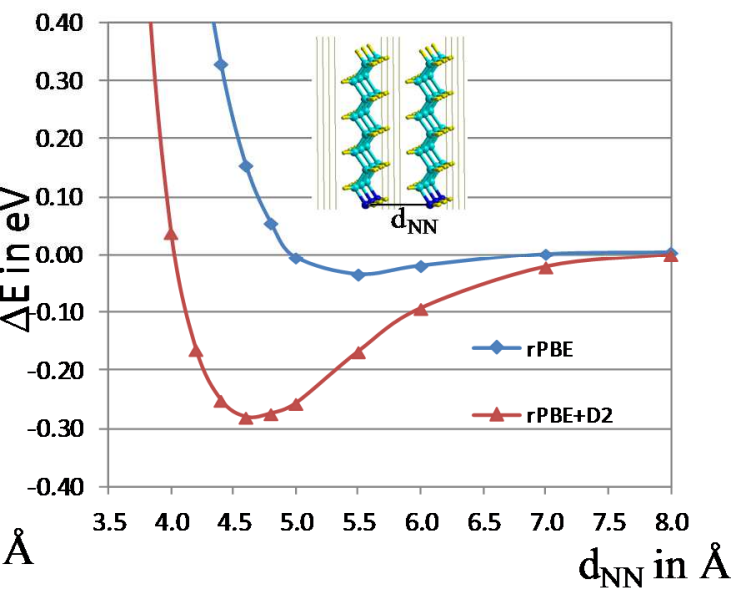

Figure 1. Energy profile of gas-phase octylamine systems. Energies are given relative to an octylamine molecule in the gas-phase, in eV. Left: profile for a dimer. Right: profile for a square 2D packed system (one molecule in the unit cell).

\subsection{Molecule-surface interaction}

The adsorption site preferred for the $\mathrm{NH}_{2} \mathrm{C}_{8} \mathrm{H}_{17}$ molecule is a quasi-top site [14], as found also for $\mathrm{NH}_{3}$ [25]. Concerning the molecule orientation on the surface, previous calculations on Au/thiol SAMs show that the alkyl chains are not perpendicular to the surface but tilted [26-28], and is also the case in $\mathrm{Ag} /$ thiol systems [29]. Our calculated value for the tilting angle $\alpha$ (see Figure 2 ) is $\sim 23 \mathrm{deg}$ (rPBE), $\sim 27 \mathrm{deg}$ (D2) with a gain in energy of $-0.33 \mathrm{eV}$ (rPBE) $-0.55 \mathrm{eV}$ (D2) with respect to the molecule perpendicular to the slab. Also, the relative orientation of the molecule with respect to the surface sites has been explored through the radial angle beta, 45 deg being the optimal angle for all the methods (see Figure 2). The system is much less sensitive to this radial angle than to the tilting angle, the gain in energy is small (0.09 eV for rPBE, $0.05 \mathrm{eV}$ for $\mathrm{rPBE}+\mathrm{D} 2)$. 

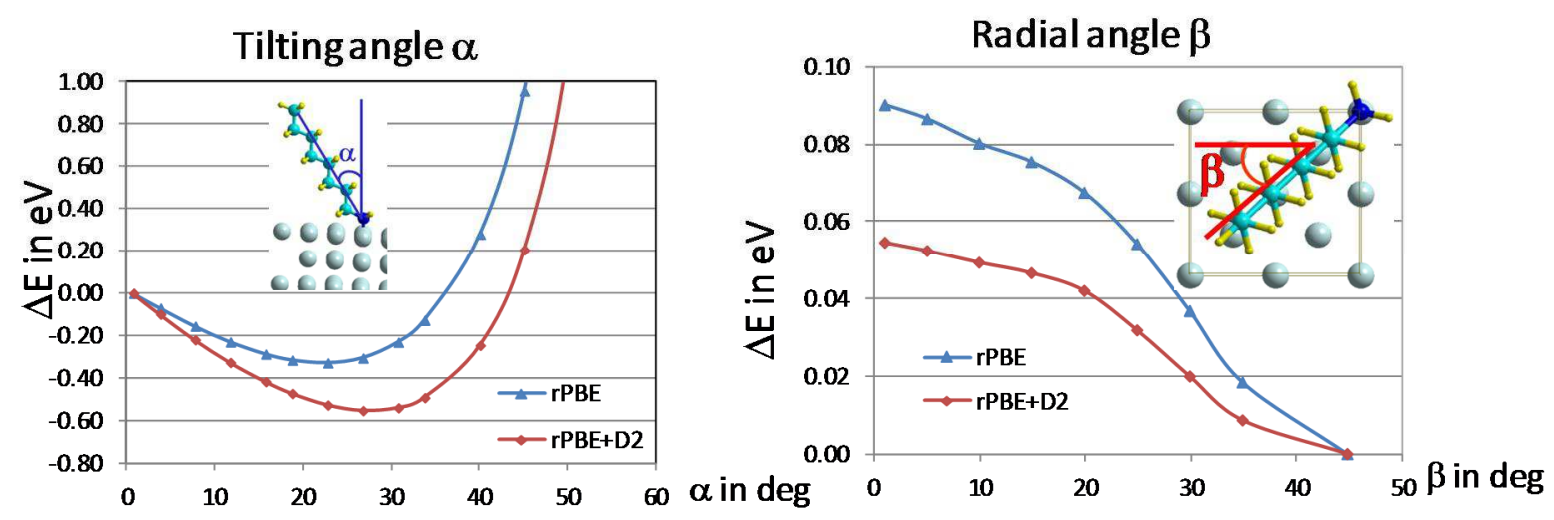

Figure 2. Energy profile for the geometry of octylamine on a $2 \times 2 \mathrm{Pt}(100)$ slab as a function of the angle. Left: tilting angle to the vertical $\alpha$ (side view) Right: radial angle $\beta$ (top view)

The coverage effect has been investigated for coverage values $\theta$ from 0.11 to 0.5 . The coverage is defined as the number of molecules per surface Pt atom as in Table 1. For all the coverages considered the adsorption occurs on a quasi-top position, the platinum atom to which the amine is bonded slightly moving upwards. The most favorable adsorption energy is found for $\theta=0.125$, and becomes less favorable as the coverage increases. For a coverage of $\theta=0.5$ the amine is almost vertical to the surface, but as the coverage decreases the tilting angle increases reaching values around $30 \mathrm{deg}$. This is an indication of the higher intermolecular interaction occurring when the molecules are close to each other (high coverage). Low coverage involves almost exclusively molecule-surface interaction due to long distances between neighboring molecules. The Pt-N distance is higher than $2 \AA$ in all cases. 

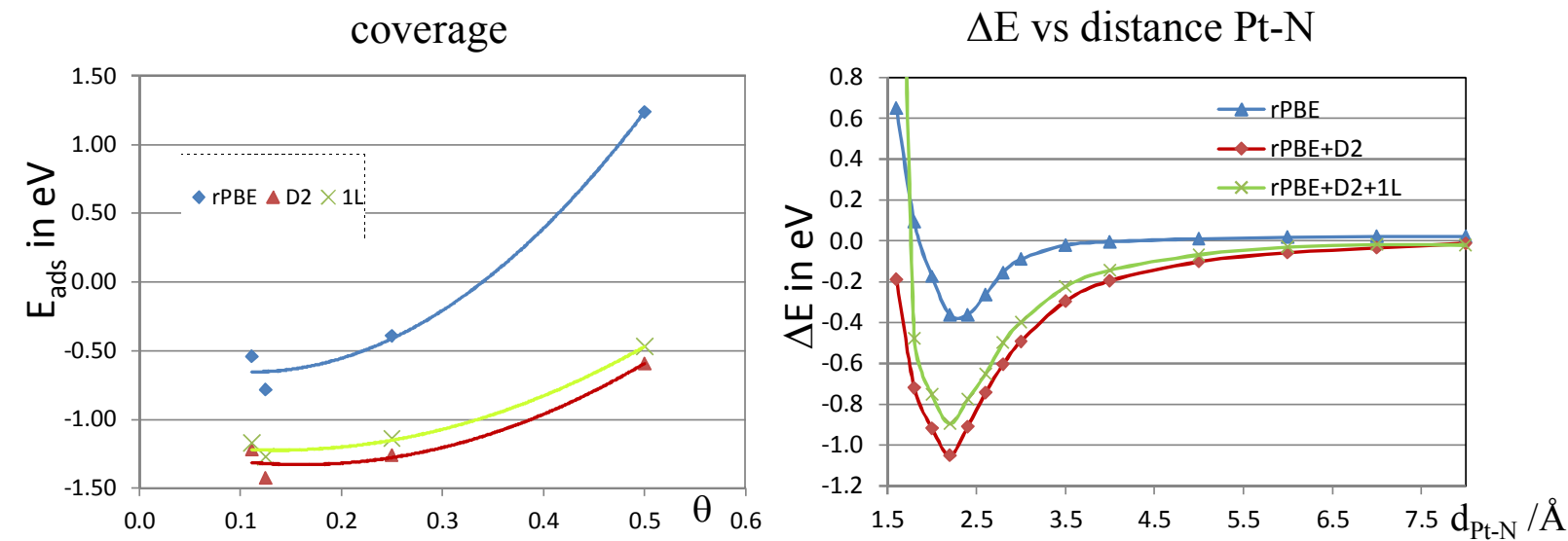

Figure 3. Energy profile for the molecule-surface interactions as a function of the coverage (left) and Pt-N distance (right). Lines are a guide for the eye.

In order to investigate the interaction between the molecule and the surface we have selected the coverage $\theta=0.25$. At this coverage the adsorption energy is quite exothermic and the neighboring molecules interact at a distance of $5.65 \AA$ which means that the adsorbate-substrate interaction is predominant. We have studied the potential energy curves for the molecule as a function of the distance with the surface, $d_{\mathrm{pt}-\mathrm{N}}$, keeping the geometry fixed. Figure 3 right displays the curve obtained. The rPBE calculations lead to a shallow minima $\left(d_{\mathrm{Pt}-\mathrm{N}}=2.2-2.4 \AA, \mathrm{E}_{\mathrm{int}}=-0.39 \mathrm{eV}\right)$. The D2 calculations result in a deeper well $\left(d_{\mathrm{pt}-\mathrm{N}}=2.2 \AA, \mathrm{E}_{\mathrm{int}}=-1.26 \mathrm{eV}\right)$. The $1 \mathrm{~L}$ calculations lie in between the curves $\left(d_{\mathrm{Pt}-\mathrm{N}}=2.2 \AA, \mathrm{E}_{\mathrm{int}}=-1.14 \mathrm{eV}\right)$, closer to the full D2 values. It can be concluded that the equilibrium distance between the octylamine and the Pt slab is around $2.2 \AA$, which indicates a physisorption. The adsorption energy is moderate, around $-1.2 \mathrm{eV}$ when considering dispersion forces and $-0.39 \mathrm{eV}$ at the rPBE level. Dispersion is therefore more important in the energetics of adsorption, which results in a significant increase from $-0.39 \mathrm{eV}$ to $-1.2 \mathrm{eV}$, than in the geometry, that results in a slightly shorter Pt-N bond upon inclusion of the Grimme correction. 
Table 1. Geometric and energetic parameters considered for the unit cells given. Coverage $\theta$, cell parameter $a$ in $\AA$, adsorption energy $\mathrm{E}_{\mathrm{ads}}$ in $\mathrm{eV}$, distance $\mathrm{Pt}-\mathrm{N}$ in and tilting angle $\chi$ in degrees.

\begin{tabular}{|c|c|c|c|c|c|c|c|c|c|}
\hline & & & rPBE & & & D2 & & $1 \mathrm{~L}$ & \\
\hline Unit cell & $\theta$ & $a$ & $\mathrm{E}_{\mathrm{ads}}$ & $d_{P t-N}(\alpha)$ & $a$ & $E_{\text {ads }}$ & $d_{P t-N}(\alpha)$ & $\mathrm{E}_{\mathrm{ads}}$ & $d_{P t-N}(\alpha)$ \\
\hline $1 \times 1$ (10 at) & 0.5 & 3.975 & 1.24 & $2.024(21.0)$ & 3.985 & -0.59 & $2.190(5.5)$ & -0.47 & $2.187(5.5)$ \\
\hline V2V2 (20 at) & 0.25 & 5.653 & -0.39 & $2.145(26.0)$ & 5.635 & -1.26 & $2.142(26.0)$ & -1.14 & $2.134(26.0)$ \\
\hline $2 \times 2$ (40 at) & 0.125 & 8.00 & -0.78 & $2.151(29.7)$ & 7.969 & -1.42 & $2.111(28.0)$ & -1.27 & $2.130(29.8)$ \\
\hline V3V3 (45 at) & 0.111 & 8.48 & -0.54 & $2.150(32.3)$ & 8.453 & -1.22 & $2.118(30.7)$ & -1.17 & $2.127(31.9)$ \\
\hline
\end{tabular}

\subsection{Orientation}

As shown in Section 3.1, Figure 1, the gas-phase packing of octylamine molecules leads to a significant stabilization of the square 2D arrangement. In the ordered superstructure the interaction between neighboring molecules depend on the relative orientation. Figure 4 displays the relative orientation of two neighboring octylamines in the $\mathrm{x}$ and $\mathrm{y}$ directions. It can be observed that they are not equivalent since they involve different $\mathrm{H}-\mathrm{H}$ interactions. Along the $y$ direction, octylamine molecules are oriented in such a way that the hydrogen atoms in the chains face those of the neighboring molecule. Instead, there is no such orientation when molecules arrange along the $x$ direction and hydrogens are not face to face.

A closer look at the geometries enables understanding that this difference comes from a different geometrical arrangement (Figure 4). In these figures, the $y$ alignment is clearly different than the $x$ one as regards $\mathrm{H}-\mathrm{H}$ interactions. In order to gain more information on the role of the relative orientation of the two chains, we have considered polymers of octylamine in the $\mathrm{x}$ and $\mathrm{y}$ directions separately. Tests on the propilamine dimer at MP2 level are displayed in Figure S3. They indicate that the $x$ dimer is slightly more stable than the $y$ one $(-0.054 \mathrm{eV}$ for $\mathrm{x},-0.04 \mathrm{eV}$ for $\mathrm{y})$, and the equilibrium 
intermolecular distance is longer for the former ( $4.7 \AA$ vs $4.4 \AA$ ). The same system calculated at the rPBE+D2 level gives $x$ and $y$ dimers almost isoenergetic $(-0.05 \mathrm{eV})$ with $\mathrm{d}_{\mathrm{NN}}$ distaces at equilibrium of $4.8 \AA$ ( $\mathrm{x}) 4.4 \AA$ (y). This indicates a slight overestimation of the $y$ interactions compared to the $\mathrm{x}$ ones in the D2 approach used.

Figure S4 shows the energetic profile of 1D polymers along $x$ and $y$ directions as a function of the distance between neighboring molecules $\mathrm{d}_{\mathrm{NN}}$. It can be observed that the stabilization in the alignment along $y$ direction is higher than in the $\mathrm{x}$ direction which is in part due to the method as we have seen above. Comparing now the $2 D$ pattern to the addition of $\mathrm{x}$ and y polymers (Figure S3 right) the result is a net stabilization of the $2 \mathrm{D}$ square pattern with respect to the addition of $\mathrm{x}$ plus $\mathrm{y}$, almost inexistent for rPBE but significant when using the D2 approach. Although the method used overstabilizes the interactions in the $y$ direction, the overall stabilization of the $2 \mathrm{D}$ pattern compared to the sum of the polymers is significant. This result highlights the key role of second neighbors in the stabilization of the squared superstructure.

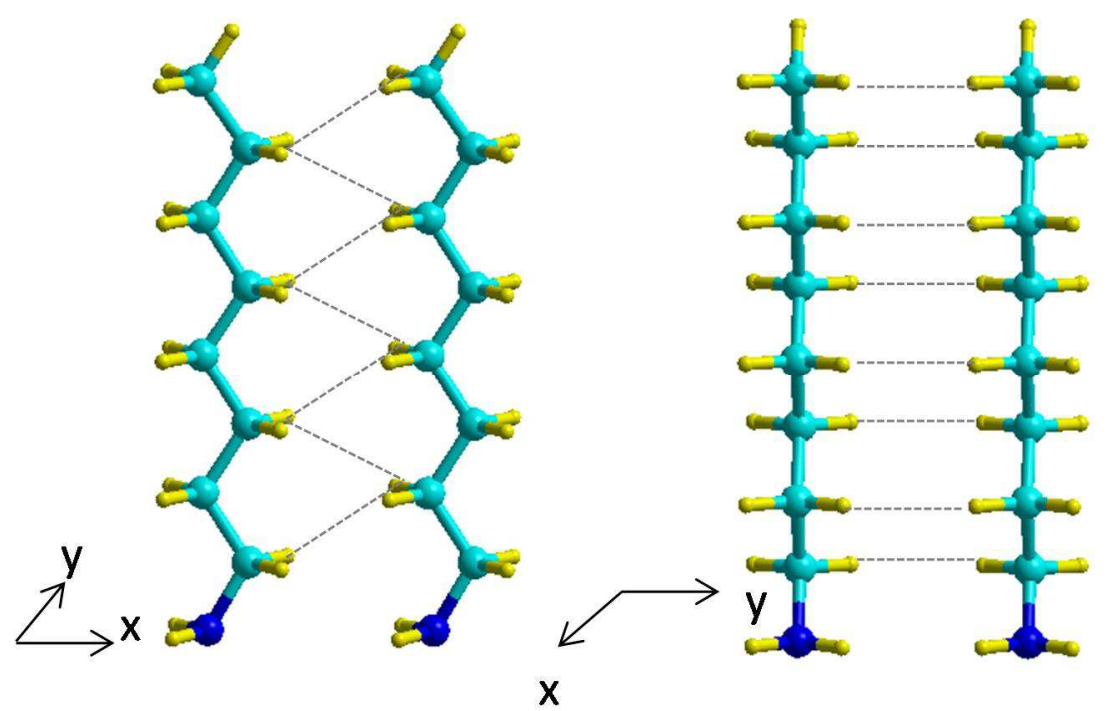

Figure 4. Octylamine molecules along $x$ (left) and $y$ (right) direction in a square 2D model superstructure. Interacting hydrogen atoms are connected by dotted lines. $\mathrm{H}$ atoms in yellow, $\mathrm{C}$ in cyan, $\mathrm{N}$ in blue. 
The relative orientation of the neighbors plays thus a key role in stabilizing extended systems. In order to have a better insight on the energetics we have calculated the energy profile of a gas-phase octylamine dimer as a function of the relative orientation of the two molecules. It can be seen in Figure $\mathrm{S} 4$ that the molecules rotated $\chi=80 \mathrm{deg}$ in the $\mathrm{z}$-axis is the most favourable situation. This orientation is similar to the one obtained for the $y$-polymer that would correspond to $\chi=90 \mathrm{deg}$ (Figure 4, right). On the other side, having the molecules face to face, $\chi=0 \mathrm{deg}$, is less favourable and corresponds to the x-polymer in Figure 4 left. Note that both the rPBE and D2-corrected series give very similar energetic profiles.

\subsection{Bonding}

In order to understand the effects of these geometrical arrangements at the bonding level, we have carried out $\mathrm{NCl}$ calculations at the $\mathrm{rPBE}+\mathrm{D} 2$ level. Figure 5 shows the isocontour plots of the $\mathrm{NCl}$ index of the octylamine molecule polymer model along the $x$ and $y$ directions. Note that $\mathrm{NCl}$ is very little affected by the level of calculation at a given geometry: the same features are found with the MP2 curves of the propylamine dimer, see Figure 5 right. The reduced density $s$ vs $\rho$ diagrams for the three systems can be found in Figure S5. 


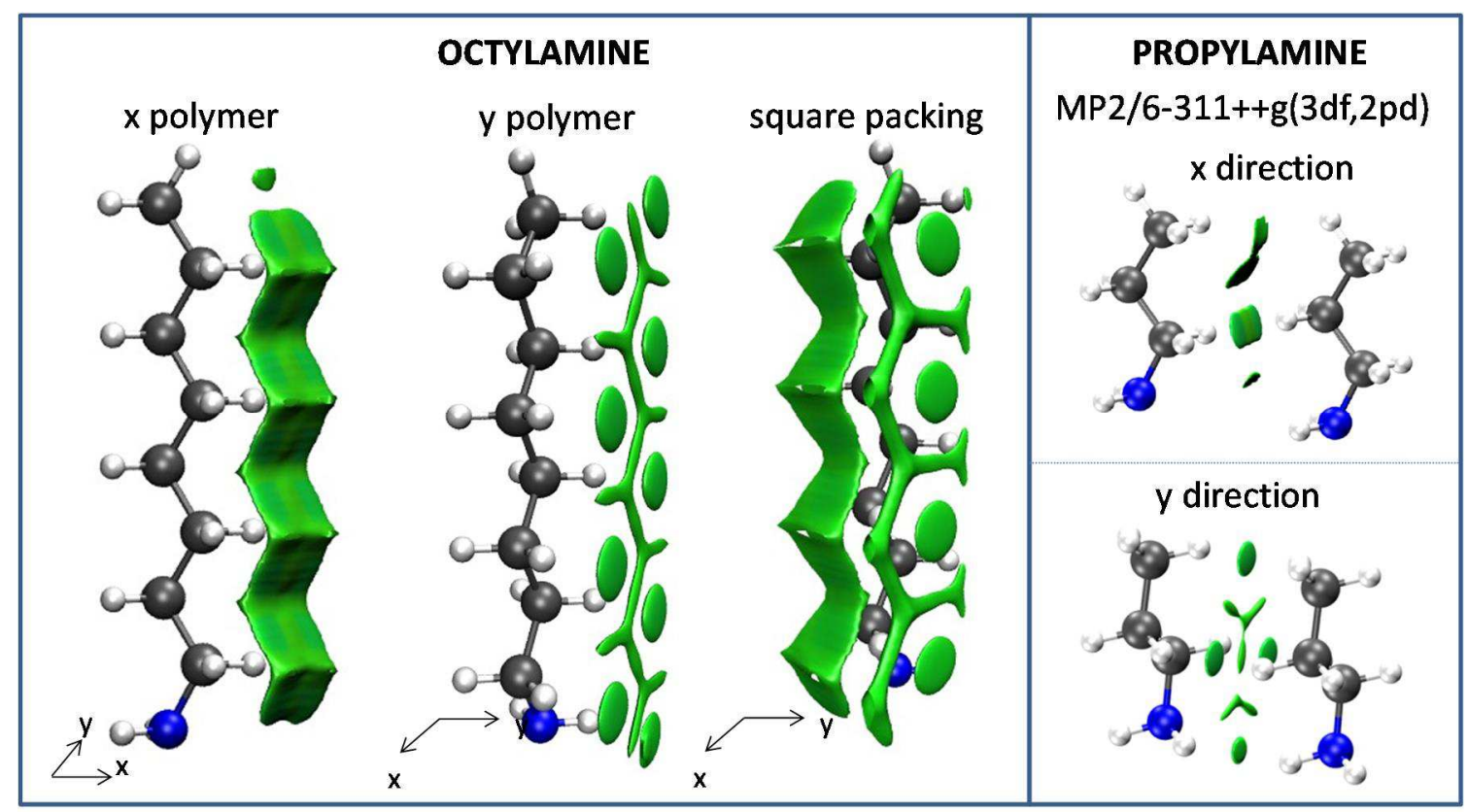

Figure 5: $\mathrm{NCl}$ isocountours (in green) for the interaction of octylamines in periodic systems as found for the the $x$ and $y$ polymers and the square 2D packed system.

As expected, $\mathrm{NCl}$ isosurfaces are anisotropic, differentiating between delocalized and localized interactions along $\mathrm{x}$ and $\mathrm{y}$ directions respectively. The symmetric arrangement along the $y$ direction gives rise to localized $\mathrm{H}-\mathrm{H}$ interactions between them, whereas delocalized $\mathrm{H}-\mathrm{CH}_{2}$ interactions appear along the $x$ direction. The directionality of $\mathrm{H}-\mathrm{H}$ interactions is characterized within the $\mathrm{NCl}$ approach by the small, round shape of the regions and the strength by the relatively large accumulation of electron density. Weak interactions such as the ones found in the $x$ polymer give rise to interaction regions, rather than localized $\mathrm{NCl}$ domains, and present a relatively low electron density. In both cases, the colour of the $\mathrm{NCl}$ isosurfaces reveals the weak non-bonding nature of all the interactions, which agrees with the need to resort to D2 corrections to account for them.

Moreover, the stabilizing effect of $\mathrm{H}-\mathrm{H}$ interactions along the $y$ direction raises a fundamental problem in crystal packing, which is still an open question. These interactions are usually assumed to 
give rise to steric clashes, and contribute to the destabilization of the crystal. However, topological approaches such as QTAIM point towards their stabilizing nature.

The H-H BCPs were first interpreted by Cioslowski and Mixon in kekulene [30] and ortho-substituted biphenyls [31] and assigned to "non-bonded repulsive contacts", a view supported by later studies with different energy partitions [32, 33]. QTAIM atomic energies, on the other hand, predict a stabilization caused by $\mathrm{H}-\mathrm{H}$ contacts of up to $10 \mathrm{kcal} / \mathrm{mol}$ in the general case of polybenzenoid molecules [34] . It should be noted that the weakness of dihydrogen interactions and the arbitrariness in the choice of energy partition precludes determining how much these interactions contribute to the total binding energy of a crystal, so it would be extremely difficult to determine in an unbiased manner the strength and character of these interactions, except in those cases where the total stabilization is to a good approximation, only given by those interactions. This is the case of the y polymer. Being hydrogen interactions the main source of interaction in the y direction (very little dispersion is observed), the attractive interaction observed in this polymer can only be attributed to $\mathrm{H}-\mathrm{H}$ interactions.

In the case of octylamine 2D square packing, the stabilizing nature of $\mathrm{H}-\mathrm{H}$ interactions in the $y$ direction is supported by i) the presence of clear $\mathrm{NCl}$ maps regions instead of atom-atom contacts and ii) the energetic curves obtained after inclusion of the D2 Grimme correction.

From the visual analysis of $\mathrm{NCl}$ isosurfaces of the linear polymers and the 2D arrangement (Figure 5), the $x$ and $y$ components are perfectly recovered, along with an extra region between interactions along $x$ and $y$ direction. This interaction between diagonal elements of the arrangement, may be identified as the responsible of its relative stability. In other words, the additive effect of the diagonal interactions is related to packing. It is interesting to note that once again, these interactions are dispersive-like, which explains why they are so difficult to track in solids. The use of $\mathrm{NCl}$ index allows therefore revealing the packing interactions in a visual way and provides a new tool to analyse dispersion interactions in extended systems. 


\section{Conclusion}

A combined periodic DFT and $\mathrm{NCl}$ approach has been used to describe the geometry, energetics and bonding in a model of self-assembled monolayer octylamine-platinum. The dispersion effects are found to play a major role in the molecular packing energetics and a moderate effect in the adsorption geometry. We have been able to identify the stabilizing nature of dispersive $\mathrm{H}-\mathrm{H}$ interactions in packing thanks to the $\mathrm{NCl}$ index. The $\mathrm{NCl}$ analysis combined with dispersive corrections in standard DFT calculations are thus powerful tools to understand molecular packing in extended systems.

\section{Acknowledgements}

This work was performed using HPC resources from GENCI- CINES/IDRIS (Grant 2013- x2013082131, Grant 2014- x2014082131) and the CCRE-DSI of Université P. M. Curie. Dr. B. Diawara is warmly acknowledged for the Modelview visualization program. This work undertaken partially in the framework of CALSIMLAB is supported by the public grant AND-11-LABX-0037-07 overseen by the

French National Research Agency (ANR) as part of the "Investissements d'Avenir" program (reference: ANR-11-IDEX-0004-02).

\section{References}

[1] J.C. Love, L.A. Estroff, J.K. Kriebel, R.G. Nuzzo, G.M. Whitesides, Self-Assembled Monolayers of Thiolates on Metals as a Form of Nanotechnology, Chem. Rev. 105 (2005) 1103-1170.

[2] J.P. Folkers, P.E. Laibinis, G.M. Whitesides, Self-assembled monolayers of alkanethiols on gold: comparisons of monolayers containing mixtures of short- and long-chain constituents with methyl and hydroxymethyl terminal groups, Langmuir 8 (1992) 1330-1341.

[3] C. Salzemann, C. Petit, Influence of Hydrogen on the Morphology of Platinum and Palladium Nanocrystals, Langmuir 28 (2012) 4835-4841. 
[4] M. Chen, B. Wu, J. Yang, N. Zheng, Small Adsorbate-Assisted Shape Control of Pd and Pt Nanocrystals, Advanced Materials 24 (2012) 862-879.

[5] L. Ferrighi, Y.-x. Pan, H. Grönbeck, B. Hammer, Study of Alkylthiolate Self-assembled Monolayers on Au(111) Using a Semilocal meta-GGA Density Functional, J. Phys. Chem. C 116 (2012) 7374-7379.

[6] S. Grimme, Semiempirical GGA-type density functional constructed with a long-range dispersion correction, J. Comp. Chem. 27 (2006) 1787.

[7] S. Grimme, J. Antony, S. Ehrlich, H. Krieg, A consistent and accurate ab initio parametrization of density functional dispersion correction (DFT-D) for the 94 elements H-Pu, J. Chem. Phys. 132 (2010) 154104.

[8] G. Mercurio, E.R. McNellis, I. Martin, S. Hagen, F. Leyssner, S. Soubatch, J. Meyer, M. Wolf, P. Tegeder, F.S. Tautz, K. Reuter, Structure and Energetics of Azobenzene on Ag(111):

Benchmarking Semiempirical Dispersion Correction Approaches, Phys. Rev. Lett. 104 (2010) 036102.

[9] C. Melis, L. Colombo, A. Mattoni, Self-Assembling of Poly(3-hexylthiophene), J. Phys. Chem. C 115 (2011) 576-581.

[10] P.N. Abufager, J.G. Solano Canchaya, Y. Wang, M. Alcami, F. Martin, L. Alvarez Soria, M.L. Martiarena, K. Reuter, H.F. Busnengo, Theoretical study of the structure of self-assembled monolayers of short alkylthiolates on $\mathrm{Au}(111)$ and $\mathrm{Ag}(111)$ : the role of induced substrate reconstruction and chain-chain interactions, Phys. Chem. Chem. Phys. 13 (2011) 9353-9362.

[11] J.G.S. Canchaya, Y. Wang, M. Alcami, F. Martin, H.F. Busnengo, Study of the interaction between short alkanethiols from ab initio calculations, Phys. Chem. Chem. Phys. 12 (2010) 7555-7565.

[12] F. Tielens, V. Humblot, C.-M. Pradier, Exploring the reactivity of mixed $\omega$-functionalized undecanethiol self-assembled monolayers-A DFT study, Int. J. Quantum Chem. 108 (2008) 1792-1795.

[13] W. Andreoni, A. Curioni, H. Grönbeck, Density functional theory approach to thiols and disulfides on gold: Au(111) surface and clusters, Int. J. Quantum Chem. 80 (2000) 598-608.

[14] N. Aguilera-Porta, M. Calatayud, C. Salzemann, C. Petit, Understanding How in Situ Generated Hydrogen Controls the Morphology of Platinum Nanoparticles, J. Phys. Chem. C 118 (2014) 9290-9298.

[15] J.P. Perdew, K. Burke, M. Ernzerhof, Generalized gradient approximation made simple, Phys. Rev. Lett. 77 (1996) 3865-3868.

[16] Y.K. Zhang, W.T. Yang, Comment on "Generalized Gradient Approximation Made Simple", Phys. Rev. Lett. 80 (1998) 890.

[17] G. Kresse, J. Hafner, Ab initio molecular dynamics for liquid metals, Phys. Rev. B 47 (1993) 558.

[18] G. Kresse, J. Hafner, Ab initio molecular-dynamics simulation of the liquid-metal-amorphoussemiconductor transition in germanium, Phys. Rev. B 49 (1994) 14251.

[19] P.E. Blochl, Projector augmented-wave method, Phys. Rev. B 50 (1994) 17953-17979.

[20] G. Kresse, D. Joubert, From ultrasoft pseudopotentials to the projector augmented-wave method, Phys. Rev. B 59 (1999) 1758-1775.

[21] E.R. Johnson, S. Keinan, P. Mori-Sanchez, J. Contreras-Garcia, A.J. Cohen, W.T. Yang, Revealing Noncovalent Interactions, J. Am. Chem. Soc. 132 (2010) 6498-6506.

[22] J. Contreras-García, M. Calatayud, J.-P. Piquemal, J.M. Recio, Ionic interactions: Comparative topological approach, Comput. Theor. Chem. 998 (2012) 193-201.

[23] A. Otero-de-la-Roza, M.A. Blanco, A.M. Pendas, V. Luana, Critic: a new program for the topological analysis of solid-state electron densities, Comput. Phys. Comm. 180 (2009) 157166.

[24] W. Humphrey, A. Dalke, K. Schulten, VMD - Visual Molecular Dynamics, J. Molec. Graphics 14 (1996) 33-38. 
[25] G. Novell-Leruth, A. Valcarcel, A. Clotet, J.M. Ricart, J. Perez-Ramirez, DFT Characterization of Adsorbed NHx Species on Pt(100) and Pt(111) Surfaces, J. Phys. Chem. B 109 (2005) 1806118069.

[26] F. Tielens, D. Costa, V. Humblot, C.M. Pradier, Characterization of omega-functionalized undecanethiol mixed self-assembled monolayers on $\mathrm{Au}(111)$ : A combined polarization modulation infrared reflection-absorption spectroscopy/X-ray photoelectron spectroscopy/periodic density functional theory study, J. Phys. Chem. C 112 (2008) 182-190.

[27] F. Tielens, V. Humblot, C.-M. Pradier, M. Calatayud, F. Illas, The stability of binary SAMs formed by omega-acid and alcohol functionalized thiol mixtures, Langmuir 25 (2009) 9980.

[28] F. Tielens, V. Humblot, C.M. Pradier, Exploring the reactivity of mixed omega-functionalized undecanethiol self-assembled monolayers - A DFT study, Int. J. Quantum Chem. 108 (2008) 1792-1795.

[29] D. Torres, P. Carro, R.C. Salvarezza, F. Illas, Evidence for the Formation of Different Energetically Similar Atomic Structures in $\mathrm{Ag}(111)-(\mathrm{V} 7 \mathrm{xV} 7)-\mathrm{R} 19.1 \mathrm{CH}_{3} \mathrm{~S}$, Phys. Rev. Lett. 97 (2006) 226103.

[30] J. Cioslowski, S.T. Mixon, W.D. Edwards, Weak bonds in the topological theory of atoms in molecules, J. Am. Chem. Soc. 113 (1991) 1083-1085.

[31] J. Cioslowski, S.T. Mixon, Topological properties of electron density in search of steric interactions in molecules: electronic structure calculations on ortho-substituted biphenyls, J. Am. Chem. Soc. 114 (1992) 4382-4387.

[32] J.D. Dunitz, A. Gavezzotti, Molecular Recognition in Organic Crystals: Directed Intermolecular Bonds or Nonlocalized Bonding?, Angew. Chem. Intl. Ed. 44 (2005) 1766-1787.

[33] J. Poater, M. Solà, F.M. Bickelhaupt, Hydrogen-Hydrogen Bonding in Planar Biphenyl, Predicted by Atoms-In-Molecules Theory, Does Not Exist, Chem. Eur. J. 12 (2006) 2889-2895.

[34] C.F. Matta, J. Hernández-Trujillo, T.-H. Tang, R.F.W. Bader, Hydrogen-Hydrogen Bonding: A Stabilizing Interaction in Molecules and Crystals, Chem. Eur. J. 9 (2003) 1940-1951. 